\title{
Complex and Severe Amiodarone-Induced Pleuro- Pulmonary Toxicity
}

\author{
Salem Bouomrani S 1,2*, Salsabil Dabboussi ${ }^{3,4}$, Nesrine Regaïeg ${ }^{1}$, Nesrine Begacem¹, Maher Béji ${ }^{1,4}$ \\ ${ }^{1}$ Department of Internal medicine. Military Hospital of Gabes, Tunisia \\ ${ }^{2}$ Sfax Faculty of Medicine. University of Sfax, Tunisia
}

${ }^{3}$ Department of Pneumology. Military Hospital of Tunis, Tunisia

${ }^{4}$ Tunis Faculty of Medicine. Tunis El Manar University, Tunisia

Received: 眥 September 26, 2018; Published: 畊 October 03, 2018

*Corresponding author: Salem Bouomrani, Department of Internal medicine, Military Hospital of Gabes, Tunisia

\begin{abstract}
Introduction: Pulmonary complications related to amiodarone have become exceptional with the doses currently used; their frequency is estimated at $1.6-2 \%$. Acute or subacute pneumonia is the most classic manifestation. Pleural involvement under amiodarone is exceptional and unusual. We report an original observation of triple toxicity to amiodarone with concomitant pulmonary parenchymal, pleural and hepatic involvement and which was spontaneously resolved.

Case Report: 71-years-old patient, hypertensive and diabetic type 2, without degenerative complications, treated with amiodarone for four years for atrial fibrillation, was hospitalized because of severe acute pneumopathy evolving for ten days. Biology revealed moderate cytolytic hepatitis (ASAT at $130 \mathrm{IU} / \mathrm{l}$ and ALAT at $243 \mathrm{IU} / \mathrm{l}$ ) without cholestasis or liver failure. Chest X-ray and thoracic CT showed bilateral and diffuse interstitial infiltrates, severe bilateral pneumonitis of both upper lobes, diffuse patchy infiltrates and ground-glass opacity, and bilateral pleural effusion. Abdominal ultrasound and CT showed moderate homogeneous and hyperdense hepatomegaly without focal lesions. The infectious, immunological, and tumoral investigations were negative. The hypothesis of drug toxicity was retained, and the evolution was rapidly favorable after stopping amiodarone with disappearance of respiratory complaints, normalization of liver tests, and progressive radiological cleansing. Chest radiography and thoracic CT scan were substantially normal at six months.
\end{abstract}

Conclusion: Amiodarone-induced complex pulmonary toxicity with parenchymal and pleural involvement remains exceptional and not well known by clinicians. Regular clinical and radiological monitoring are recommended to detect and manage them in time, and improve the prognosis given the risk of irreversible fibrosis evaluated at 5-7\% of cases.

Keywords: Amiodarone; Pneumonitis; Pleuritis; Amiodarone Pulmonary Toxicity; Hepatitis; Toxicity

Abbreviations: ASAT: Aspartate Aminotransferase; ALAT: Alanine Aminotransferase; Ana: Antinuclear Antibodies; P-Anca: Perinuclear Antineutrophil Cytoplasmic Antibodies; C-Anca: Cytoplasmic Antineutrophil Cytoplasmic Antibodies; Anti-Lkm1: AntiLiver/Kidney Microsome Antibodies; Anti-M2: Anti-Mitochondrial Antibodies M2 Subtype

\section{Introduction}

Amiodarone is a class III antiarrhythmic agent widely used for the treatment of ventricular and supraventricular rhythm disorders $[1,2]$; its spectrum of toxicity is wide: thyroid, heart, lung, eye, skin, liver, gastrointestinal tract, and central nervous system [3]. All these effects are mediated by the mitochondrial toxicity of the drug and its lipophilicity. This toxicity is not dose-dependent, can occur even very early, and men seems to be more exposed than women

[4]. The current incidence of amiodarone pneumonia is estimated at $1.6-2 \%[5,6]$. His most classic presentation is acute or subacute pneumonia, while pleural involvement is exceptional $[3,7]$ and often described as an unusual manifestation of amiodarone-induced pneumopathy $[7,8]$; similarly, acute hepatitis under amiodarone is very rare $(<3 \%)[6]$. We are reporting an original observation of triple toxicity to amiodarone with concomitant bilateral pleural, pulmonary parenchymal and hepatic involvement. 


\section{Case Report}

71-years-old patient, hypertensive and diabetic type 2, without degenerative complications, treated with amiodarone for four years for atrial fibrillation, was hospitalized because of severe acute pneumopathy evolving for ten days. The examination found apyretic patient, dyspneic (polypnea at 26 cycles/min and spontaneous oxygen saturation at $82 \%$ ) with diffuse crepitant rales, bilateral pleuritis, and moderate hepatomegaly. The electrocardiogram showed slower atrial arrhythmia (mean frequency at $90 / \mathrm{min}$ ) and the cardiac enzymes were not elevated. Chest X-ray showed bilateral and diffuse interstitial infiltrates (Figure 1). Thoracic CT revealed severe bilateral pneumonitis of both upper lobes (Figure 2) with diffuse patchy infiltrates and ground-glass opacity (Figure 3), and bilateral pleural effusion (Figure 4). Abdominal ultrasound and CT showed moderate homogeneous and hyperdense hepatomegaly without focal lesions.

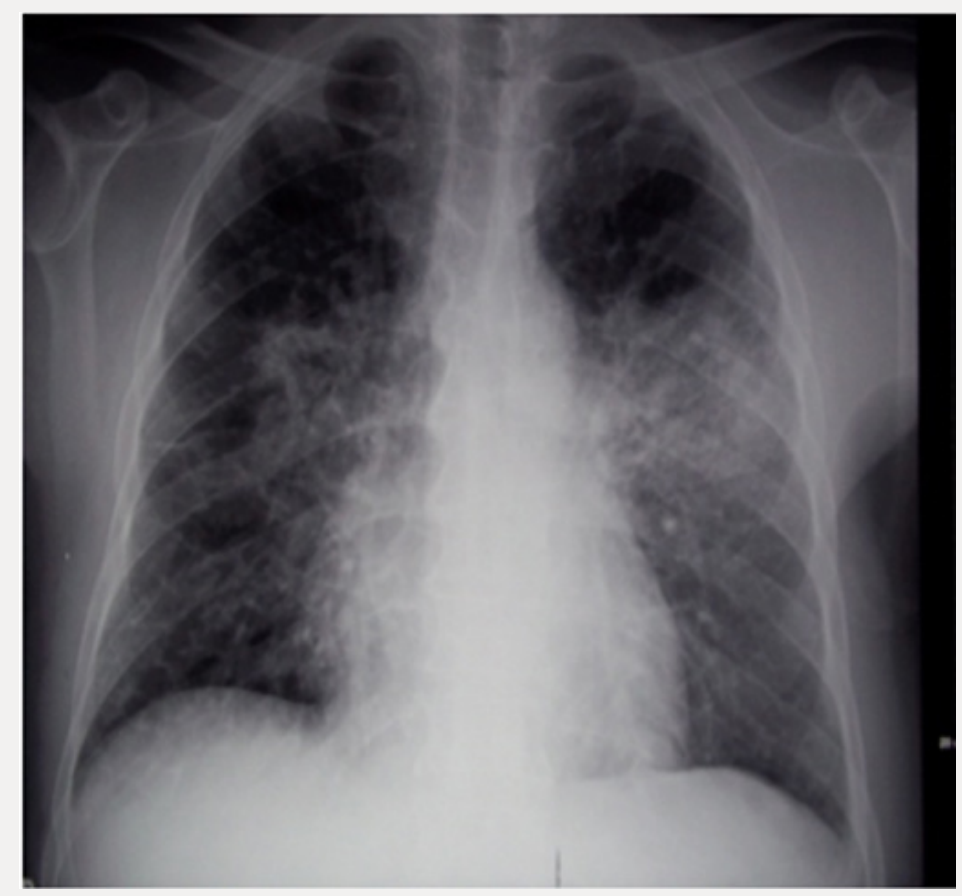

Figure 1: Chest X-ray (posteroanterior view): bilateral and diffuse interstitial infiltrates.

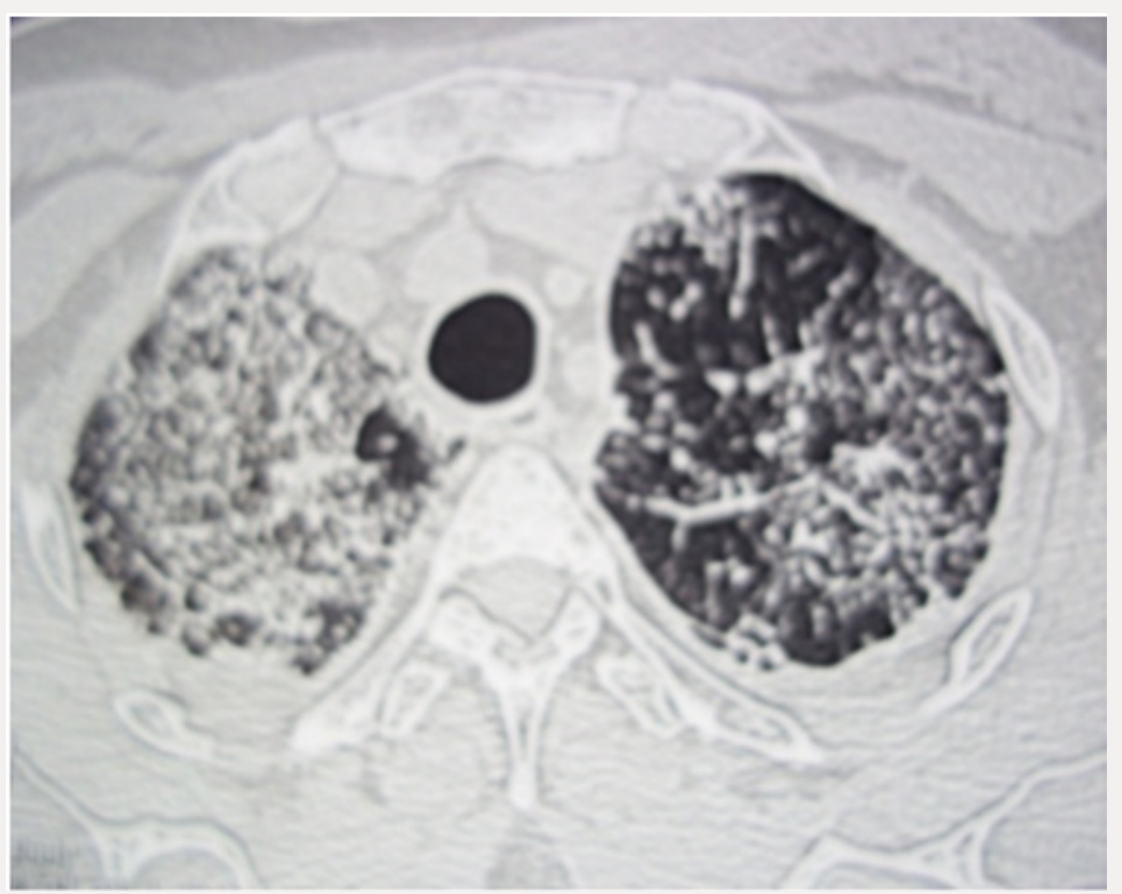

Figure 2: Thoracic CT in axial section without contrast: severe bilateral pneumonitis of both upper lobes. 


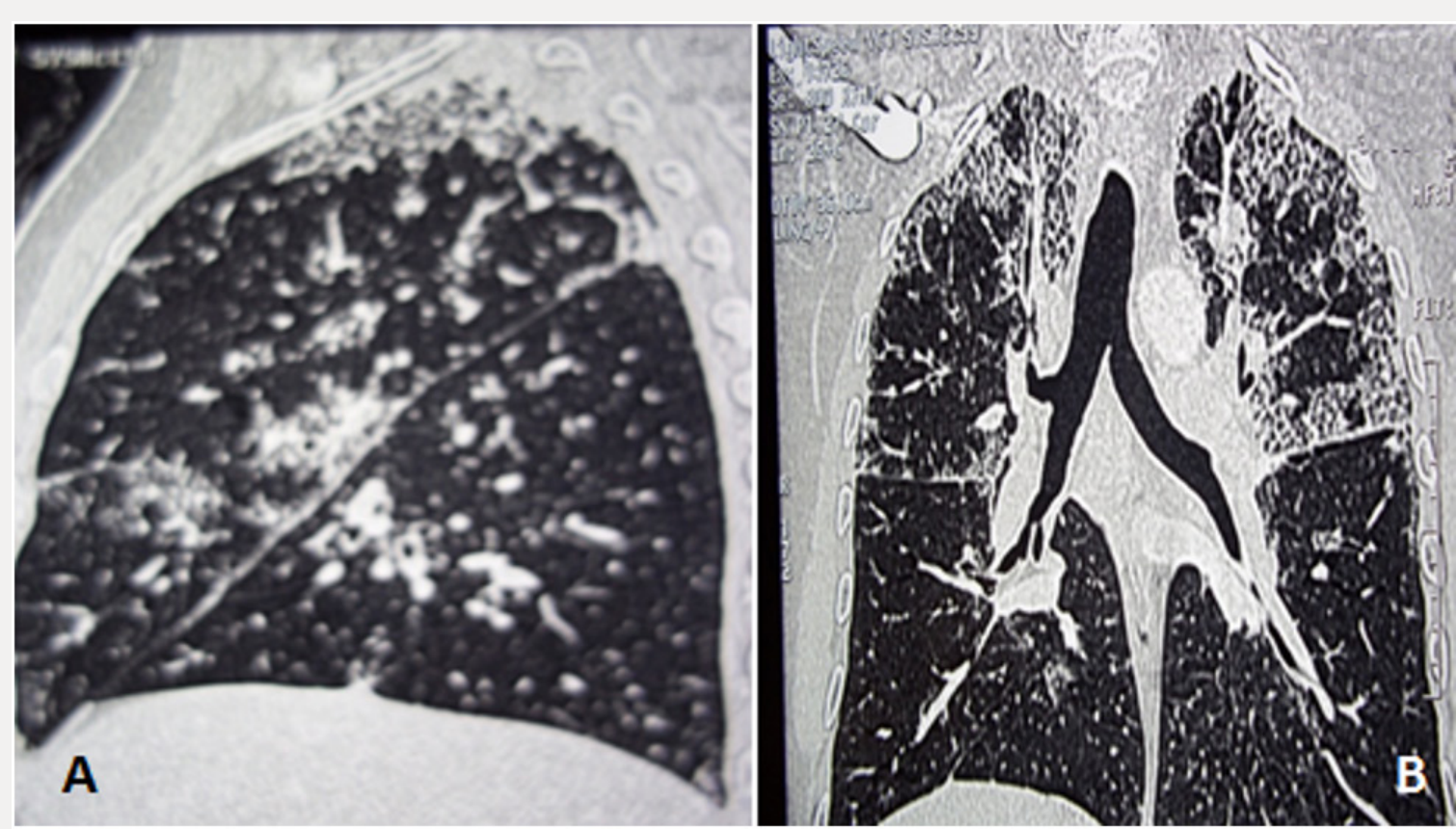

Figure 3: Thoracic CT with three-dimensional reconstruction (parasagittal (A) and coronal (B) views): diffuse patchy infiltrates and ground-glass opacity.

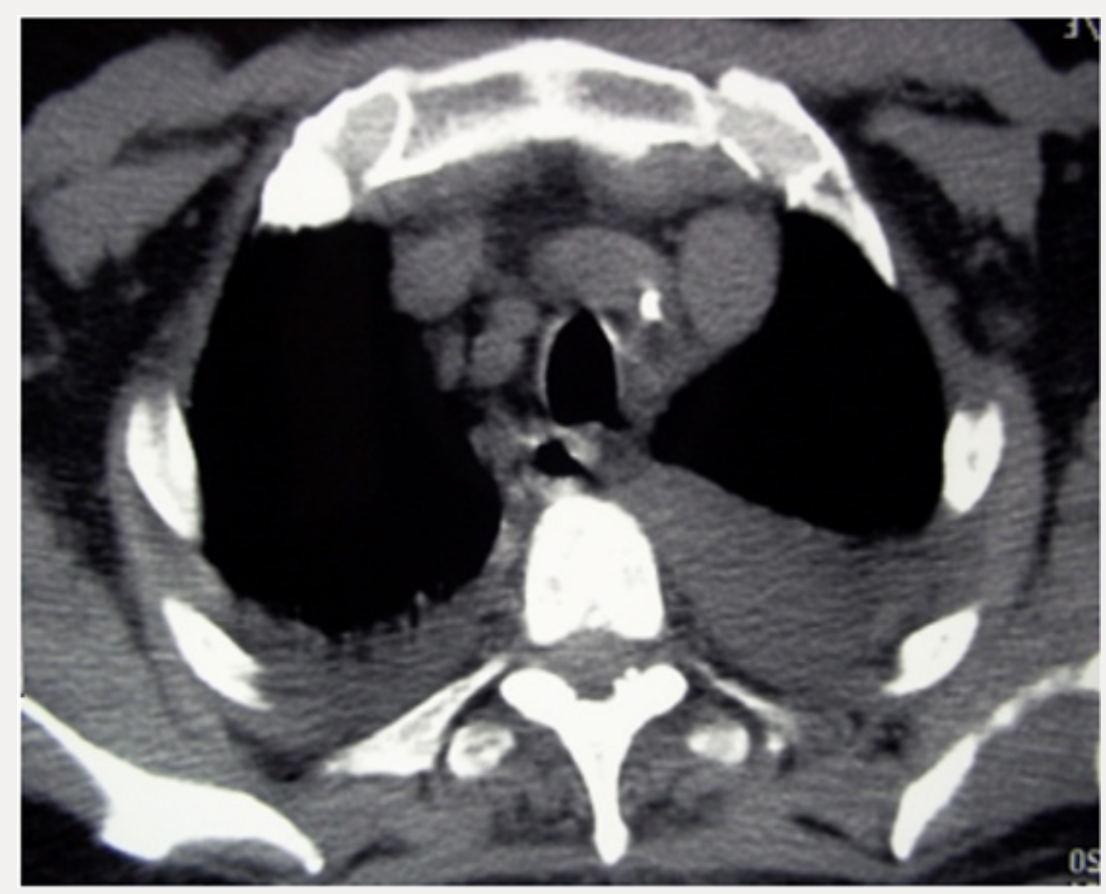

Figure 4: Thoracic CT in axial section without contrast (mediastinal window): bilateral pleural effusion.

Biology revealed moderate cytolytic hepatitis (ASAT at 130 IU/l and ALAT at 243 IU/l) without cholestasis or liver failure. The other basic biological tests were within normal limits: Leucocytes, platelet, hemoglobin, erythrocyte sedimentation rate, C-reactive protein, creatinine, ionogram, glycemic parameters, muscle enzymes, electrophoresis of serum proteins, and urine analysis. The infectious (bacteriological, viral and tuberculous), immunological
(ANA, p-ANCA, c-ANCA, anti LKM1, anti M2 antibodies) and tumor investigation was negative. The diagnosis of drug toxicity was retained, and the evolution was rapidly favorable after stopping amiodarone with disappearance of respiratory complaints, normalization of liver tests, and progressive radiological cleansing. Chest radiography and thoracic CT scan were substantially normal at six months. 


\section{Discussion}

Although rarely observed, toxic pulmonary involvement caused by amiodarone is a major cause of discontinuation of this treatment $[1,7,9]$. Classically assessed at $5-7 \%[2,10]$, amiodaroneinduced pneumopathy appears to be clinically overestimated $[5,6]$. Indeed, in the large controlled and double-blind study, including 3439 patients receiving daily $400 \mathrm{mg}$ or less amiodarone, pulmonary toxicity was noted only in $1.6 \%$ [5]. This pulmonary toxicity of amiodarone has been markedly reduced using lower doses of the drug but can occur with any dose [3]. Clinically, amiodarone-induced pneumopathy may manifest itself as: cough, chest discomfort, often progressive dyspnea, chest pain, and more rarely hemoptysis or acute respiratory failure in the exceptional forms of intra-alveolar haemorrhage or acute respiratory distress syndrome $[2,9,11]$. However, it can remain totally infra-clinical (insidious), and would only be found in pulmonary imaging $[2,9,11]$. Radiologically, this toxic pneumopathy may be in the form of: diffuse interstitial lung disease, acute or chronic organizing pneumonia, eosinophilic pneumonia, single or multiple nodules or mass-like lesions, desquamative interstitial pneumonia, and diffuse alveolar haemorrhage $[3,11,12]$. These lesions can be rapidly progressive [2] with the risk of irreversible pulmonary fibrosis $[2,9,12]$. Pleural effusion/pleurisy remains exceptional during amiodarone pulmonary toxicity $[3,7,8]$. It is classically bilateral and exudative $[8,13]$. It is rarely isolated [7], most often associated with parenchymal pulmonary toxicity or other toxic organ damage $[8,13]$, and may exceptionally be, as in our observation, the first sign indicative of this toxicity $[8,13]$. Hepatic injury with amiodarone is rare and often unpredictable. Its frequency is estimated at $15-30 \%$ for elevated liver enzymes and less than 3\% for acute hepatitis [6]. It can be irreversible in $50 \%$ of cases and even lead to severe forms of steatohepatitis with cirrhosis and portal hypertension [6]. Similarly, pulmonary involvement can also progress to irreversible fibrosis in 5 to $7 \%$ of cases [14] with a mortality estimated at $9 \%$ [2]. Treatment is based on discontinuation of the drug and, in severe cases, systemic corticosteroids for 4 to 12 months; the initial dose is $40-60 \mathrm{mg} /$ day followed by a gradual decline [3]. Corticosteroid therapy allows rapid improvement of pulmonary gas exchange and reversibility of radiological abnormalities [10]. Relapse after discontinuation of corticosteroid therapy has been reported mainly in obese subjects. Early detection of amiodaroneinduced pulmonary toxicity significantly improves its prognosis which can be fatal $[2,9,12](50 \%$ of cases if acute respiratory distress syndrome [2]), and clinical recommendations are currently well established for this [5,6].

\section{Conclusion}

The pulmonary toxicity of amiodarone has become rare with the use of lower doses; it can happen even early enough and with any dose. Regular clinical, radiological and biological monitoring makes it possible to detect and take these complications in time. Our observation is characterized by the association of a triple toxicity with amiodarone: pulmonary, hepatic, and pleural which is exceptional, and by its spontaneous resolution. It is necessary to think of the iatrogenic cause due to amiodarone in front of any bilateral pleurisy that is not proven in patient under amiodarone.

\section{References}

1. Lee KY, Oh S, Choi YJ, Oh SH, Yang YS, et al. (2013) Activation of autophagy rescues amiodarone-induced apoptosis of lung epithelial cells and pulmonary toxicity in rats. Toxicol Sci 136(1): 193-204.

2. Papiris SA, Triantafillidou C, Kolilekas L, Markoulaki D, Manali ED (2010) Amiodarone: review of pulmonary effects and toxicity. Drug Saf 33(7): 539-558.

3. Wolkove N, Baltzan M (2009) Amiodarone pulmonary toxicity. Can Respir J 16(2):43-48.

4. Huang X, Yang Y, Zhu J, Gao X, Wang G, et al. (2009) Clinical applications and acute hepatotoxicity of intravenous amiodarone. J Int Med Res 37(6): 1928-1936.

5. Sunderji R, Kanji Z, Gin K (2000) Pulmonary effects of low dose amiodarone: a review of the risks and recommendations for surveillance. Can J Cardiol 16(11): 1435-1440.

6. Goldschlager N, Epstein AE, Naccarelli GV, Olshansky B, Singh B, et al. (2007) A practical guide for clinicians who treat patients with amiodarone: 2007. Heart Rhythm 4(9): 1250-1259.

7. McNeil KD, Firouz Abadi A, Oliver W, Zimmerman PV (1992) Amiodarone pulmonary toxicity--three unusual manifestations. Aust N Z J Med 22(1): 14-18.

8. Uong V, Nugent K, Alalawi R, Raj R (2010) Amiodarone-induced loculated pleural effusion: case report and review of the literature. Pharmacotherapy 30(2): 218.

9. Van Cott TE, Yehle KS, DeCrane SK, Thorlton JR (2013) Amiodaroneinduced pulmonary toxicity: case study with syndrome analysis. Heart Lung 42(4): 262-266.

10. Vasić NR, Milenković BA, Pešut DP, Stević RS, Jovanović DM (2014) Drug induced lung disease--amiodarone in focus. Med Pregl 67(9-10): 334337.

11. Hudzik B, Polonski L (2012) Amiodarone-induced pulmonary toxicity. CMAJ 184(15): 819.

12. Kang IS, Kim KJ, Kim Y, Park SH (2014) The diagnostic utility of chest computed tomography scoring for the assessment of amiodaroneinduced pulmonary toxicity. Korean J Intern Med 29(6): 746-753.

13. Gonzalez Rothi RJ, Hannan SE, Hood CI, Franzini DA (1987) Amiodarone pulmonary toxicity presenting as bilateral exudative pleural effusions. Chest 92(1): 179-182.

14. Camus P, Martin WJ, Rosenow EC (2004) Amiodarone pulmonary toxicity. Clin Chest Med 25(1): 65-75. 
(C) (P) This work is licensed under Creative Commons Attribution 4.0 License

To Submit Your Article Click Here: $\quad$ Submit Article

DOI: 10.32474/LOJPCR.2018.01.000106

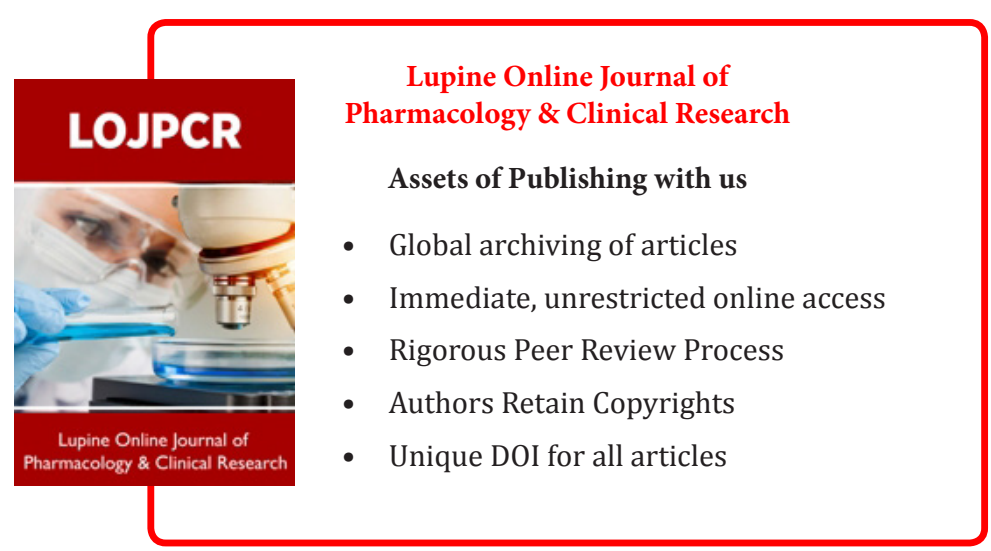

